Original Paper http://ajol.info/index.php/ijbcs http://indexmedicus.afro.who.int

\title{
Modélisation du séchage solaire de fèves de cacao par le Réseau de Neurones Artificiel
}

\author{
Daouda KARIDIOULA ${ }^{1,2 *}$, Djedjro Clément AKMEL ${ }^{2}$, Nogbou Emmanuel ASSIDJO $^{2}$ et \\ Albert TROKOUREY ${ }^{1}$ \\ ${ }^{I}$ Université Félix Houphouet Boigny (UFHB) de Cocody, UFR SSMT, Laboratoire de Chimie - Physique ; 22 \\ BP 582 Abidjan 22, Côte d'Ivoire. \\ ${ }^{2}$ Institut National Polytechnique Félix Houphouët Boigny (INP-HB) de Yamoussoukro, Laboratoire des \\ Procédés Industriels de Synthèse de l'Environnement et des Energies Nouvelles (LAPISEN), \\ BP 1093, Côte d'Ivoire. \\ *Auteur correspondant ; E-mail: karidioulad@yahoo.fr; Tel : (+225) 07259670
}

\section{RESUME}

Les fèves de cacao sont des produits de plus en plus convoités par l'industrie agro-alimentaire et cosmétique du fait de leurs nombreuses vertus thérapeutiques. Ces produits sont généralement à hautes humidités ou à humidités intermédiaires d'où l'intérêt de leur stabilisation par le séchage. L'obtention d'un cacao bien séché qui satisfait les exigences du client nécessite la maîtrise de ce processus. Dans le but de mieux appréhender le séchage solaire, une modélisation a été effectuée. Un modèle mathématique de l'évolution de la teneur en eau des fèves de cacao a été réalisé à l'aide d'un réseau de neurones artificiel. Le réseau de neurones le mieux adapté est le perceptron multicouche avec deux neurones sur la couche d'entrée, deux neurones sur la couche cachée et un neurone sur la couche de sortie. L'application de ce modèle a révélé un écart minime entre valeurs expérimentales et valeurs prédites des teneurs en eau (X). Le coefficient de détermination $\mathrm{R}^{2}$ de la régression linéaire entre valeurs observées et valeurs prédites des teneurs en eau $(\mathrm{X})$ a été 0,99 . Ainsi le modèle a présenté de bonnes prévisions et a servi à la simulation du séchage solaire de fèves de cacao.

(C) 2018 International Formulae Group. All rights reserved.

Mots clés : Séchage solaire, fèves de cacao, modélisation, simulation, réseau de neurones artificiel (RNA).

\section{Modeling the solar drying of cocoa beans by the Artificial Neural Network}

\begin{abstract}
Cocoa beans are increasingly coveted by the food industry. These products are generally at high humidity or at intermediate humidities, hence the advantage of their stabilization by drying. Obtaining a well dried cocoa beans that meet the customer's requirements requires mastering this process. In order to better understand the solar drying, a modeling was carried out. A mathematical model of the evolution of the water content of cocoa beans was carried out using an artificial neural network. The most suitable neural network is the multilayer perceptron with two input neurons, two neurons on the hidden layer and a neuron on the output layer. The application of this model revealed a small difference between experimental values and predicted values of water contents $(\mathrm{X})$. The coefficient of determination $\mathrm{R}^{2}$ of the linear regression between observed values and
\end{abstract}


predicted values of water contents $(\mathrm{X})$ was 0.99 . Thus, the model presented good predictions and was used to simulate the solar drying of cocoa beans.

(C) 2018 International Formulae Group. All rights reserved.

Keywords: Solar drying, cocoa beans, modelization, simulation, artificial neural network (RNA).

\section{INTRODUCTION}

Le séchage est l'une des méthodes courante de conservation de la plupart des produits agroalimentaires, par diminution de leur teneur en eau jusqu'à des valeurs résiduelles où le développement de tout micro-organisme est inhibé. Le stockage est alors possible dans des conditions ambiantes. Depuis les premiers travaux de LEWIS (Lewis, 1921) et de SHERWOOD (Sherwood, 1929) cette technique a fait l'objet de nombreuses communications scientifiques et demeure encore de nos jours un domaine de recherche privilégié.

L'opération de séchage doit être réalisée en optimisant la quantité de chaleur nécessaire pour obtenir un produit de qualité avec une dépense globale minimale d'énergie. La technique qui consiste à faire circuler les gaz chauds, issus de la combustion d'énergie fossile, directement sur les produits joue sur la qualité du produit séché (Madhlopa et al., 2002). Cependant, le séchage électrique reste un procédé très onéreux et non utilisable dans les secteurs ruraux des pays en développement.

Le développement des énergies renouvelables, tout particulièrement l'énergie solaire, a été sérieusement envisagée car il est une solution qui intègre les différentes problématiques liées au séchage, à savoir la flambée des prix et la pénurie de carburants utilisés comme source d'énergie (Busunia et Abe., 2001). Cette technique est une alternative raisonnable, en particulier dans les pays en développement dont le degré d'ensoleillement est élevé tout au long de l'année et où l'utilisation des méthodes traditionnelles s'impose dans bien de domaines.

L'amélioration rationnelle de cette technique passe par la maîtrise du comportement des produits lors du séchage.

Cette étude va consister à modéliser, puis à simuler le phénomène du séchage de fèves de cacao dont l'importance et l'intérêt économique n'est plus à démontrer en Côte d'Ivoire, premier producteur mondial de cacao.

\section{MATERIEL ET METHODES}

\section{Matière biologique}

Le matériel biologique utilisé dans l'étude est le cacao (Forastero) de Toumbokro, village de la région de Yamoussoukro. Le cacao a subi toutes les étapes post-récolte dont le décabossage en vue d'extraire les fèves et la fermentation de celles-ci.

\section{Méthodes expérimentales}

L'étude expérimentale a consisté à sécher sur une claie les fèves fermentées en relevant l'évolution de la masse à des intervalles de temps réguliers. Ceci grâce à une balance électronique de marque SARTORIUS BL 1500 (précision 1g). Nous avons supposé que les temps de pesée (quelques secondes) n'ont aucune influence sur la cinétique de séchage (Narendra et Parthasarathy, 1990). Ainsi, les taux d'humidité $(X)$ ont été obtenus par la formule suivant :

$$
X=\frac{m_{e}}{m_{s}} \quad \text { Avec } \mathrm{m}_{\mathrm{e}}=\mathrm{m}-\mathrm{m}_{\mathrm{s}}
$$

Où $\mathrm{m}_{\mathrm{e}}$ : masse d'eau

$\mathrm{m}$ : masse totale des fèves

$\mathrm{m}_{\mathrm{s}}$ : masse sèche ou anhydre des fèves 
Le séchage a été effectué en période sèche dans les conditions ambiantes, conditions dans lesquelles la température, la vitesse, et l'humidité relative de l'air de séchage évoluent très peu. En outre, un thermomètre, un humidimètre et un anémomètre ont été utilisés pour le contrôle de la stabilité de ces différents paramètres. Les valeurs moyennes obtenues pour ces paramètres sont :

- Température $31^{\circ} \mathrm{C}$;

- Vitesse $1 \mathrm{~m} / \mathrm{s}$;

- Humidité relative $66 \%$.

L'expérience de séchage a été répétée 21 fois dans les mêmes conditions.

\section{Modélisation du processus de séchage}

La modélisation du processus de séchage solaire des fèves de cacao a été réalisée en utilisant la méthode des réseaux de neurones artificiels. Les logiciels EXCEL 2007 et MATLAB Release 14 (MATLAB7) ont été utilisés pour la modélisation.

\section{Choix des paramètres}

\section{Paramètres de sortie}

Le choix des paramètres de sortie dépend des grandeurs que nous désirons paramétrer. Ici, il s'agit de la teneur en eau des fèves de cacao $(\mathrm{X})$ à chaque instant du séchage.

\section{Paramètres d'entrée}

Le choix des paramètres d'entrée est une étape essentielle de la mise en œuvre de la technique. Cette étape permet d'optimiser le calcul et de réduire les erreurs afin d'obtenir le meilleur résultat possible. Les paramètres d'entrée sont sélectionnés en fonction de leur pertinence, de leur redondance et de leur disponibilité. Dans le cadre de cette étude, seul l'aspect temporel du processus a été retenu d'où le choix des paramètres suivants : t et $X_{t-1}$ qui sont respectivement le temps et la teneur en eau au temps précédent. Ce choix imposait un système récurrent.

\section{Structure du réseau de neurones artificiel}

Dans cette étude, l'architecture de base utilisée est le perceptron multicouche (PMC) avec trois types de couches à savoir: entrée, cachée et celle de sortie. Le réseau utilisé ici est caractérisé par deux (2) neurones dans la couche d'entrée (deux paramètres) et un (1) neurone dans la couche de sortie (un paramètre). Ainsi, le nombre de neurones dans la couche cachée est varié de 1 à 10 de sorte à atteindre l'architecture optimale.

\section{Modèle mathématique}

Le modèle mathématique dégagé est la sortie du neurone de la couche de sortie. Cette sortie est le résultat du calcul de tous les neurones travaillant en parallèle. Il faudra ainsi faire ressortir chaque résultat (la sortie de chaque neurone) et les prendre tous en compte. Ce modèle est donc la combinaison des sorties des neurones de la couche cachée. Il est important de noter que les calculs ont nécessité d'abord une normalisation des différentes valeurs de $t$ et $X_{t-1}$ à partir de l'équation (2) :

$$
Z=Z_{\min }+\frac{\left(z_{\max }-z_{\min }\right) *\left(h_{\max }-h_{\min }\right)}{\left(z_{\max }-z_{\min }\right)}
$$

Avec: $\mathrm{Z}_{\min }=-1$ et $\mathrm{Z}_{\max }=1 ; \mathrm{h}_{\min }$ et $\mathrm{h}_{\max }$ respectivement les valeurs minimale et maximale de $\mathrm{t}$ ou $\mathrm{X}$ et $\mathrm{Z}$ valeur normalisée de t ou X.

\section{Valeurs de sortie des neurones de la couche} cachée

La fonction de transfert utilisée pour cette couche est la fonction tangente hyperbolique (tanh) et les valeurs obtenues sont normalisées.

\section{Valeur de sortie du neurone de la couche de sortie}

La fonction de transfert sur cette couche est une fonction linéaire simple et les valeurs obtenues sont également normalisées. Une fois ces valeurs normalisées obtenues, on peut atteindre les valeurs finales des teneurs en eau en utilisant la relation suivante : 
$\mathrm{X}=\mathrm{X}_{\min }+\frac{\left(X_{\text {norm }}-Z_{\min }\right) *\left(X_{\max }-X_{\min }\right)}{\left(z_{\max }-Z_{\min }\right)}$

Avec $: Z_{\min }=-1 ; Z_{\max }=1 ; X_{\max }=1$;

$X_{\min }=0,07005503$; on a finalement :

$X=0,464972485 * X_{\text {norm }}+0,535027515$

avec, $X_{\text {norm }}=Y^{\prime}$

\section{Performance des modèles de prédiction développés}

Les modèles obtenus sont utilisés pour prédire le processus de séchage des fèves de cacao.

Le caractère de l'ajustement approprié a été apprécié par le coefficient de détermination $\left(\mathrm{R}^{2}\right)$. Ce coefficient mesure la qualité de l'ajustement de l'équation d'estimation de régression. Lorsque $\mathrm{R}^{2}$ est proche de 1 dans le cas d'une régression simple, l'ajustement entre les valeurs expérimentales et prédites est meilleur (Feinberg, 1996). Ce coefficient $\left(\mathrm{R}^{2}\right)$ est exprimé comme suit:

$$
R^{2}=\frac{\sum_{i}^{N}\left(y_{p r e ́ d, i}-\bar{y}_{p r e ́ d, i}\right)^{?}}{\sum_{i}^{N}\left(y_{\text {exp,i }}-\bar{y}_{\text {exp,i }}\right)^{2}}
$$

Avec :

$\mathrm{y}_{\text {préd,i }}$ et $\mathrm{y}_{\text {exp,i }}$ respectivement les valeurs calculées et expérimentales pour $\mathrm{i}=1, \ldots, \mathrm{N}$; et $\bar{y}_{\text {préd, } i \bar{i}}$ et $\bar{y}_{\exp , i}$ la moyenne des valeurs expérimentales et prédites.

Une autre approche consiste à déterminer la moyenne de la somme des carrés des écarts (MSE) entre $\mathrm{y}_{\text {exp,i }}$ et $\mathrm{y}_{\text {préd,i }}$ c'est-à-dire :

$$
\mathrm{MSE}=\frac{1}{N} \sum_{i}^{N}\left(y_{\exp , i}-\bar{y}_{\text {préd,i }}\right)^{2}
$$

Où N représente le nombre de points expérimentaux.

\section{RESULTATS}

Modélisation du séchage par le réseau de neurones artificiel

L'architecture du réseau de neurones artificiel

Dans le Tableau 1 est consignée la performance des réseaux de neurones artificiels construits en fonction du nombre de neurones sur la couche cachée. Il s'agit, du coefficient de corrélation (R) et de l'erreur quadratique (MSE) pour les étapes d'apprentissage et de test.

Les capacités de calcul d'un réseau de neurones résident dans les connexions qui existent entre les neurones. Chaque connexion est affectée d'une valeur numérique appelée poids (W).

Le Tableau 2 présente les poids des connexions entre les neurones de la couche cachée et ceux de la couche d'entrée et de sortie. Il est aussi donné les biais (b) des neurones de la couche cachée et celle de la sortie. La couche d'entrée ne dispose pas de biais car elle reçoit sans modification les données à traiter. Le biais d'un neurone représente l'erreur commise par le neurone lors du calcul de la valeur de sortie.

Valeurs de sortie des neurones de la couche cachée

Les valeurs de sortie des deux neurones de la couche cachée sont données par les équations (7 et 8 )

$\mathrm{Y}_{1}{ }^{\prime}=\tanh \left(\mathrm{W}_{11} * \mathrm{t}_{\text {norm }}+\mathrm{W}_{21} * \mathrm{X}_{\mathrm{t}-1 \text { norm }}+\mathrm{b}_{1}\right)$

$\mathrm{Y}_{2}{ }^{\prime}=\tanh \left(\mathrm{W}_{12} * \mathrm{t}_{\text {norm }}+\mathrm{W}_{22} * \mathrm{X}_{\mathrm{t}-1 \text { norm }}+\mathrm{b}_{2}\right)$

Avec:

$\mathrm{Y}_{1}$ ': Valeur à la sortie du premier neurone de la couche cachée ;

$\mathrm{Y}_{1}$ ': Valeur à la sortie du deuxième neurone de la couche cachée ;

$\mathrm{t}_{\text {norm: }}$ : Valeur normalisée du temps;

$\mathrm{X}_{\mathrm{t}-1 \text { norm}}$ : Valeur normalisée de la teneur en eau au temps ( $\mathrm{t}-1)$.

Valeur de sortie du neurone de la couche de sortie

La sortie du réseau, donne la valeur normalisée de la teneur en eau des fèves par l'équation :

$\mathrm{Y}^{\prime}=\mathrm{W}^{\prime}{ }_{11} * \mathrm{Y}_{1}{ }^{\prime}+\mathrm{W}^{\prime}{ }_{21} * \mathrm{Y}_{2}{ }^{\prime}+\mathrm{b}_{3}$

Avec $Y^{\prime}$ la valeur de la sortie du réseau. 
Tableau1: Valeurs de R et de MSE pour l'apprentissage et le test en fonction du nombre de neurones sur la couche cachée.

\begin{tabular}{lcccc}
\hline \multirow{2}{*}{$\begin{array}{l}\text { Nombre de neurones sur la } \\
\text { couche cachée }\end{array}$} & \multicolumn{2}{c}{ Apprentissage } & \multicolumn{2}{c}{ Test } \\
\cline { 2 - 5 } & $\mathbf{R}$ & MSE & R & MSE \\
\hline $\mathbf{2}$ & 0,99394545 & 0,00215632 & 0,99287052 & 0,00310956 \\
3 & $\mathbf{0 , 9 9 7 1 8 4 3 2}$ & $\mathbf{0 , 0 0 1 8 1 6 7 4}$ & $\mathbf{0 , 9 9 2 2 0 2 2 5}$ & $\mathbf{0 , 0 0 5 7 4 4 0 4}$ \\
4 & 0,99859250 & 0,00073823 & 0,97876026 & 0,00480997 \\
5 & 0,99874956 & 0,00116644 & 0,98809810 & 0,00263924 \\
6 & 0,99927292 & 0,00097519 & 0,97383013 & 0,00411938 \\
7 & 0,99941006 & 0,00045331 & 0,98020462 & 0,00861746 \\
8 & 0,99966202 & 0,00133670 & 0,97352444 & 0,01936999 \\
9 & 0,99967211 & 0,00052508 & 0,98382452 & 0,00926755 \\
10 & 0,99968570 & 0,00045325 & 0,97444473 & 0,00828071 \\
\hline & 0,99968726 & 0,00039415 & 0,97381502 & 0,00865709 \\
\hline
\end{tabular}

Tableau 2 : Poids et biais des différents neurones.

\begin{tabular}{|c|c|}
\hline Coefficients (W et b) & Valeurs \\
\hline $\mathrm{W}_{11}$ & 1,52507840 \\
\hline $\mathrm{W}_{12}$ & $-3,88626273$ \\
\hline $\mathrm{W}_{21}$ & $-0,03379816$ \\
\hline $\mathrm{W}_{22}$ & $-2,03573948$ \\
\hline $\mathrm{W}^{\prime}{ }_{11}$ & $-19,0701187$ \\
\hline $\mathrm{W}_{21}^{\prime}$ & $-9,76454077$ \\
\hline$b_{1}$ & 3,01509900 \\
\hline $\mathrm{b}_{2}$ & $-6,32170931$ \\
\hline$b_{3}$ & 8,32021948 \\
\hline \multicolumn{2}{|c|}{ 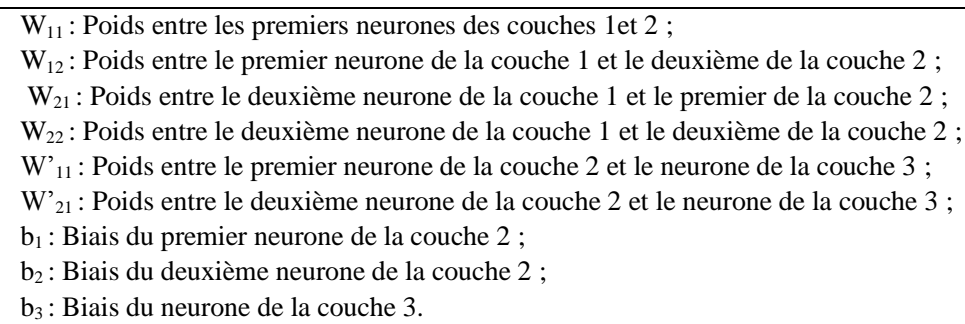 } \\
\hline
\end{tabular}

\section{DISCUSSION}

L'analyse du Tableau 1 montre que le meilleur compromis entre les coefficients de corrélation et les MSE doit obéir aux deux contraintes suivantes de manière simultanée à savoir : une MSE faible et un coefficient de corrélation (R) élevé aussi bien pour l'ensemble des apprentissages que pour l'ensemble des tests. La couche cachée à 2 neurones (Figure 1) est celle qui vérifie ces deux exigences. C'est cette architecture qui a donc été adoptée avec les caractéristiques suivantes :

- Deux (2) neurones dans la couche d'entrée ;

- Deux (2) neurones dans la couche cachée ; 
- Un (1) neurone dans la couche de sortie.

Pour cette configuration, on a une valeur de $\mathrm{R}$ de 0,99718432 et une valeur de MSE de 0,00181674 pour l'apprentissage et respectivement de 0,99220225 et de 0,00574404 pour le test.

Après la détermination de la topologie optimisée du réseau de neurones artificiel et la mise en place du modèle mathématique, l'autre phase consiste à valider cette architecture. La validation de l'architecture neuronale 2-2-1 s'est faite par l'évaluation du coefficient de détermination $\left(R^{2}\right)$ entre les valeurs prédites et les valeurs expérimentales. Cela s'est fait en traçant sur la même Figure ces différentes valeurs telles que présentées par les Figures 2 et 3. L'analyse de ces figures montre une bonne concordance entre ces valeurs, avec un coefficient de détermination $\left(\mathrm{R}^{2}\right)$ de 0,9844 proche de 1 .

\section{Validation de l'architecture neuronale optimisée}

Le modèle de réseau de neurones artificiel adopté est un compromis entre les coefficients de corrélation (R) obtenus pendant les phases d'apprentissage et de test (Chouai et al., 2000 ; Chevret, 2007). Compte tenu de tous les critères, y compris ceux du MSE, l'architecture neuronale 2-2-1 (C'est-àdire deux neurones dans la couche d'entrée, deux dans la couche cachée et un à la sortie) a été sélectionnée comme étant la meilleure topologie. Elle a été validée en utilisant des données qui n'ont pas été utilisées pendant la phase d'apprentissage.

Le processus de séchage modélisé à travers le réseau de neurones a consisté premièrement à déterminer la structure du réseau permettant d'approcher au mieux les valeurs expérimentales. Cette architecture a été obtenue après détermination des nombres de neurones des différentes couches (entrée, cachée et sortie). Où les nombres de neurones des couches d'entrée et de sortie sont respectivement les nombres de variables et de réponses; ceux de la couche cachée sont à déterminer.

Deux approches sont généralement utilisées (Le Cun et al., 1990). Dans cette étude, ce nombre a varié de 1 à 10 . La topologie neuronale 2-2-1 a été retenue comme le meilleur compromis entre les valeurs de coefficient de corrélation (R) élevée et de la moyenne de la somme des carrés des écarts (MSE) basse. Ce modèle de réseau neuronal artificiel a été validé à l'aide de données non utilisées au cours des phases d'apprentissage et de test. La valeur de $\mathrm{R}^{2}$ obtenue montre la capacité du modèle de réseau de neurones à mapper correctement le processus de séchage solaire des fèves de cacao. Les résultats obtenus sont semblables à ceux d'Akmel (2010) dans le cadre d'une étude sur la modélisation de la cinétique de séchage de fèves de cacao à travers plusieurs modèles empiriques. L'auteur a montré que le modèle logarithmique est le mieux indiqué pour traduire la cinétique de séchage des fèves, avec un R de 0,994 et une MSE de 0,0075 .

La capacité des réseaux de neurones artificiels à modéliser les processus a été soulignée dans de nombreuses études.

Assidjo et al. (2006) ont utilisé avec succès un réseau de neurones artificiel de topologie 4-4-4 pour modéliser un processus de brassage à l'échelle industrielle. Chen et Ramaswamy (2002) et Mohamed (2007) ont également utilisé avec succès un réseau de neurone artificiel respectivement, dans la modélisation de processus de transfert thermique et de cinétiques réactionnelles chimiques, dans l'industrie alimentaire.

Par conséquent, ces résultats confirment une fois de plus la capacité en général du réseau de neurones artificiel à faire des approximations des phénomènes dynamiques tels que le séchage solaire de fèves de cacao. Ainsi, il a la capacité de représenter pleinement les relations complexes non linéaires. 


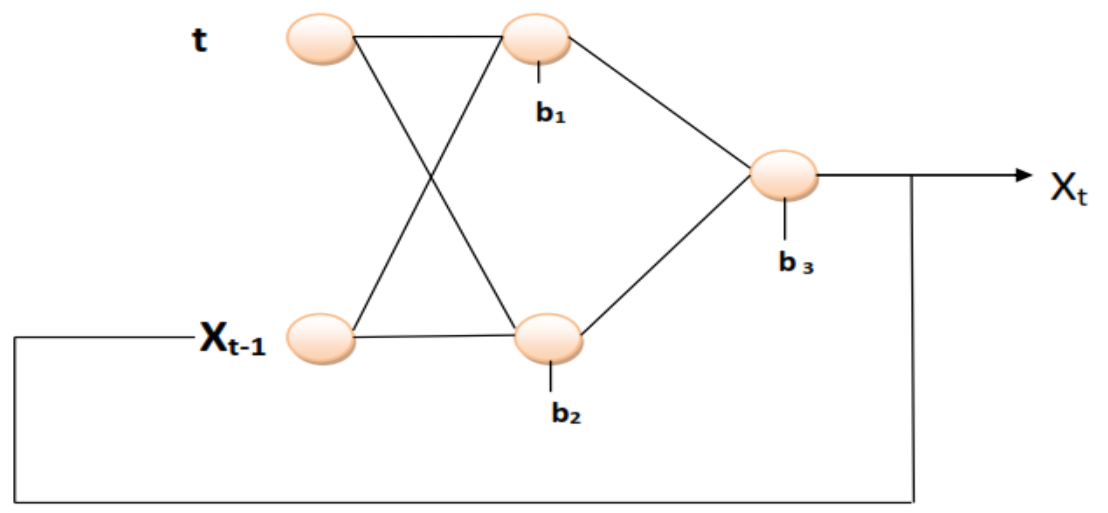

Figure 1: Schéma du réseau de neurones adopté.

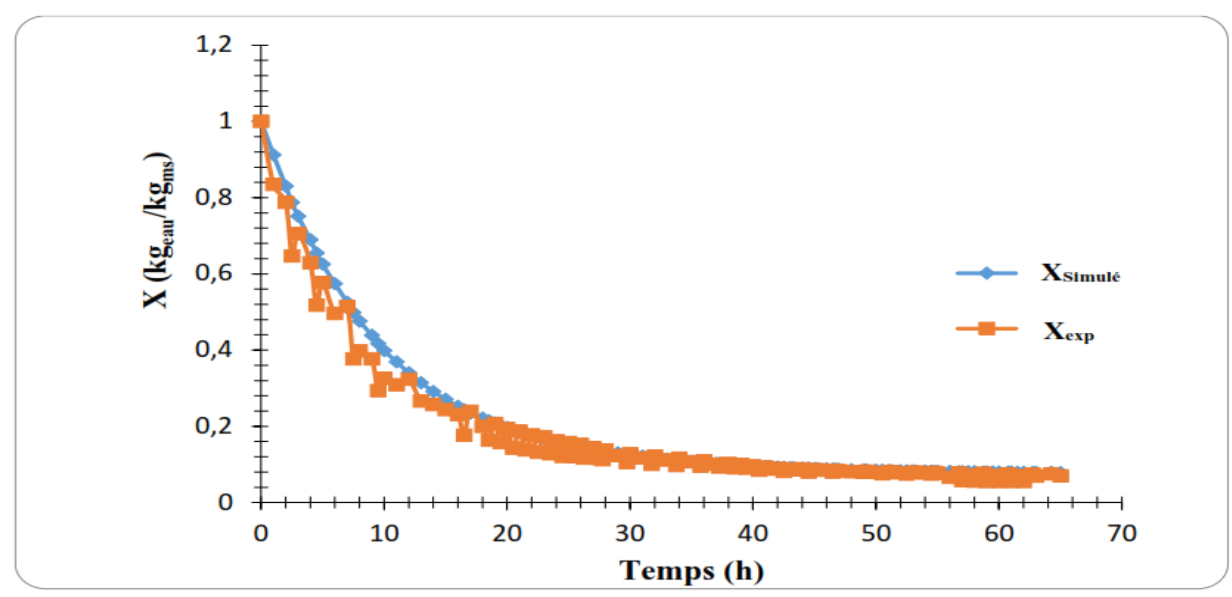

Figure 2 : Courbes simulée et expérimentale.

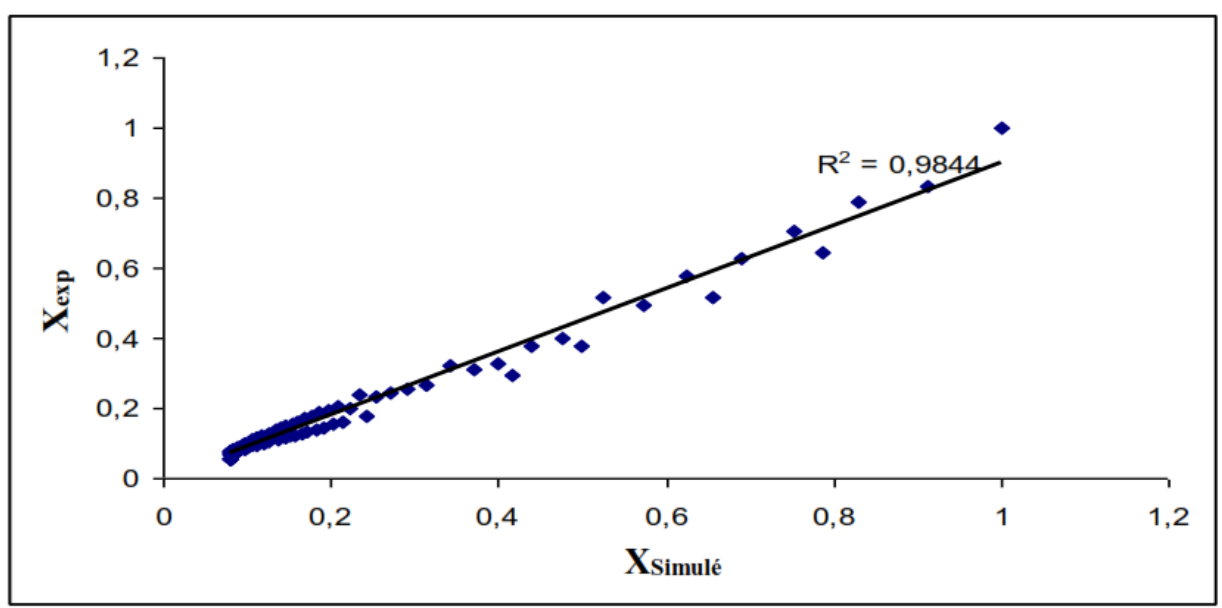

Figure 3: Corrélation entre les valeurs expérimentales et simulées. 


\section{Conclusion}

Il ressort de ce travail que l'architecture neuronale 2-2-1 est la mieux indiquée pour modéliser le processus de séchage solaire des fèves de cacao. En outre, ce modèle type "boite noire" est un bon compromis avec la réalité. Ceci valide à la fois le principe de modélisation "boite noire" et l'efficacité des réseaux de neurones dans la résolution de problèmes complexes.

\section{CONFLIT D'INTERETS}

conflit d'intérêts.

Les auteurs attestent qu'il n'y a aucun

\section{CONTRIBUTIONS DES AUTEURS}

DK est le principal investigateur de l'étude et a participé à toutes les phases du travail. NEA et DCA ont contribué à la collecte des données sur le terrain, à la mise en place du programme (Matlab) de calcul et à la correction du manuscrit. AT a assuré la supervision générale des travaux et l'interprétation approfondie des résultats.

\section{RÉFÉRENCES}

Akmel C. 2010. Séchage Solaire des fèves de cacao: étude expérimentale et modélisation de la cinétique de séchage. Thèse de doctorat, Université d'Abobo- Adjamé, Côte d'Ivoire, p.168.

Assidjo E, Yao B, Amane D, Ado G, AzzaroPantel C, Davin A. 2006. Industrial Brewery Modelling by Using Artificial Neural Network. Journal of Applied Science, 6(8): 1858-1862. DOI: Scialert.net/qredirect.php?doi= jas.2006.1858.1862\&linkid $=$ pdf

Busunia MA, Abe T. 2001. Characteristics of rough rice under natural convection. Journal of Food Engineering, 47(4):295301. DOI: https://doi.org/10.1016/ S0260-8774(00)00133-3

Chen CR, Ramaswamy HS. 2002. Modelling and optimization of variable retrot temperature (VRT) thermal processing using coupled neural networks et genetic algorithms. Journal of Food Engineering, 53(3): 209-220. DOI : https://doi.org/10.1016/S02608774(01)00159-5
Chevret S. 2007. Modèles mathématiques utilisés en médecine en France. Elsevier Masson (Science Direct), 2(16): 240244. DOI:10.1016/j.reaurg.2007.03.004

Chouai A, Cabassud M, Le Lann MV, Gourdon C, Casamatta G. 2000. Use of neural networks for liquid-liquid extraction column modelling: an experimental study. Chemical Engineering and Processing, 39(2): 171180. DOI: https://doi.org/10.1016/ S0255-2701 (99)00086-0

Feinberg M. 1996. La Validation des Méthodes d'Analyse; une Approche Chimiométrique de l'Assurance Qualité au Laboratoire, Edition Masson : Paris ; 56-57.

Le Cun Y, Denker JS, Solla SA. 1990. Optimal brain damage: Proceeding of the Neural Information Processing Systems. Sciences computers control, 2: 598-605. DOI: https://dl.acm.org

Lewis. 1921. The rate of drying of solid materials. J. Chem. Eng., 13(5): 427-432. DOI: https://doi.org/ 10.1021/ie50137a021

Madhlopa A, Jones SA, Saka JDK. 2002. A solar air heater with composite-absorber systems for food dehydration. Renewable Energy 27(1): 27-37. DOI: https://dx.doi.org/10.1016/S0960-1481 (01)00174-4

Mohamed YA. 2007. Mise en œuvre de réseaux de neurones pour la modélisation de cinétiques réactionnelles en vue de la transposition batch/continu. Thèse de Doctorat Unique. Institut National Polytechnique, Toulouse, France, pp 156.

Narendra KS, Parthasarathy K. 1990. Identification and Control of dynamical systems using Neural Networks. IEEE Trans. on Neural Networks, 1: 4- 27.

Sherwood. 1929. The drying of solids. Ind. Eng. Chem., 21(10): 12-16. DOI: 10.1021/ie50229a004. 